\title{
INCIDENCIA DE LAS ACTIVIDADES COMUNES EN LA CALIDAD DE LA COMUNICACIÓN ENTRE JEFE Y SUBORDINADO EN LAS EMPRESAS PRIVADAS
}

\author{
INFLUENCE OF SHARED TASKS IN THE QUALITY OF COMMUNICATION BETWEEN \\ EXECUTIVES AND SUBORDINATES IN PRIVATE COMPANIES
}

OMAR FABRICIO MAGUIÑA RIVERO Universidad Privada del Norte Trujillo, Perú ORCID: https://orcid.org/0000-0003-1173-7748 Correo electrónico: omarmaguina@upn.edu.pe

Ena Cecilia Obando Peralta Universidad Privada del Norte Trujillo, Perú

ORCID: https://orcid.org/0000-0001-5734-6764 Correo electrónico: ena.obando@upn.pe

Graciela Esther Reyes Pastor Universidad Privada del Norte Trujillo, Perú

ORCID: https://orcid.org/0000-0002-8206-1717

Correo electrónico: graciela.pastor@upn.pe

[Recibido: 27/04/2021 Aceptado: 17/05/2021 Publicado: 27/05/2021]

\section{RESUMEN}

Objetivo: Determinar la relación entre las actividades comunes entre el jefe y los subordinados con la calidad de la comunicación entre estos en las empresas privadas. Método: La investigación fue cuantitativa no experimental y transversal; de alcance correlacional. La población estuvo integrada por personas de la ciudad de Trujillo pertenecientes a la población económicamente activa ocupada del año 2020. La muestra fue de 384 personas pertenecientes a la población económicamente activa ocupada de la ciudad de Trujillo del año 2020. Resultados: El $43.0 \%$ de los trabajadores encuestados presentan una percepción en nivel alto de la calidad de la comunicación y el $62 \%$ de los trabajadores estiman las actividades comunes en un nivel alto. Conclusión: La relación entre las actividades comunes entre el jefe y los subordinados con la calidad de la comunicación en las empresas privadas fue altamente significativa obteniéndose un coeficiente de Spearman $(r=0,619)$, lo que demuestra que a mayor participación del jefe en actividades que realizan los subordinados, mayor calidad de la comunicación entre estos; confirmándose la hipótesis de investigación.

Palabras clave: Comunicación organizacional; síndrome de la arrogancia; el gerente fuera de la realidad.

\begin{abstract}
Objective: The objective is to determine the relationship between the shared tasks between the boss and the subordinates and the quality of communication in private companies. Method: The research was quantitative, non-experimental and correlational; the population taken into account were people belonging to the economically active employed population of the city of Trujillo in the year 2020. The sample was made up of 384 citizens of Trujillo, who belonged to the economically active employed population in the year 2020. Results: $43.0 \%$ of the surveyed workers present a high level of perception regarding the communication quality, and $62 \%$ estimate the common activities at a high level. Conclusion: The relationship between the shared tasks between the boss and the subordinates and the quality of communication was highly significative. A Spearman coefficient of $(r=0.619)$ at the level of $p<0.01$ was obtained; thus, it can be said that the higher the quality of communication, the better the development of common activities.
\end{abstract}

Keywords: Organizational communication; hubris syndrome; manager out of touch.

(0) Los autores. Este artículo es publicado por la revista Quipukamayoc de la Facultad de Ciencias Contables, Universidad Nacional Mayor de San Marcos. Este es un artículo de acceso abierto, distribuido bajo los términos de la licencia Creative Commons Atribución 4.0 Internacional (CC BY 4.0) [https://creativecommons.org/licenses/ by/4.0/deed.es] que permite el uso, distribución y reproducción en cualquier medio, siempre que la obra original sea debidamente citada de su fuente original. 


\section{INTRODUCCIÓN}

El presente estudio pretende descubrir si las actividades comunes entre jefes y subordinados puede mejorar la comunicación entre estos. Por lo que se planteó como hipótesis que, si el jefe realiza actividades comunes con los subordinados, la calidad de la comunicación entre ambos mejora. La explicación sería que la falta de entendimiento es causada por el fenómeno denominado "El gerente fuera de la realidad" o The CEO out of touch, como lo describe Roberts (2017) en su Artículo publicado en LinkedIn; fenómeno que fue identificado en políticos, como indican Owen y Davidson (2009). Adicionalmente, Owen (2012) extiende su crítica al explicar este fenómeno como aquel en que los líderes (ejecutivos, políticos o líderes religiosos o sociales) llevan o desarrollan durante el ejercicio del poder.

Las características del síndrome de la arrogancia serían:

Una propensión narcisista a ver el mundo principalmente como un escenario en el que se ejerce el poder y se busca la gloria; una predisposición a llevar a cabo acciones que parecen dar una buena imagen del individuo, es decir, con el fin de mejorar su imagen; una preocupación desproporcionada por la imagen y la presentación; una manera mesiánica de hablar sobre las actividades actuales y una tendencia a la exaltación; una identificación con la nación, o la organización hasta el punto de que el individuo considera que su perspectiva e intereses son idénticos; una tendencia a hablar en tercera persona o a utilizar el "nosotros"; excesiva confianza en el propio juicio del individuo y desprecio por los consejos o críticas de los demás; creencia exagerada en sí mismo, rayando en un sentido de omnipotencia, en lo que puede lograr personalmente; la creencia de que, en lugar de tener que rendir cuentas ante el tribunal mundano de los colegas o de la opinión pública, el tribunal ante el que responden es: la historia o Dios; una creencia inquebrantable de que en ese tribunal serán reivindicados; pérdida de contacto con la realidad; a menudo asociada a un aislamiento progresivo; e Inquietud, imprudencia e impulsividad (Owen y Davidson, 2009).

Sapolsky (2005) realizó diversos estudios en babuinos, entre ellos, el estudio que tuvo como finalidad descubrir la influencia de la jerarquía en la salud de los primates. Los resultados indican que la salud de los rangos inferiores es desventajosa, debido al estrés físico y social al que son generalmente sometidos por los que están en los rangos superiores.
La experiencia profesional y accidental de los autores muestran evidencias sobre como la comunicación en los diferentes niveles de las organizaciones, tiende a desarrollarse a través de un filtro sesgado por la pertenencia de grupo (Banaji et al., 2003). Los niveles ejecutivos tienden a percibir a los niveles inferiores con cierto grado de ineficacia y falta de esfuerzo; y por su lado, los niveles operativos e inferiores de la jerarquía organizacional tienden a ver como los niveles más altos dirigen con un sentido alejado de la realidad (Alvesson y Spicer, 2016). Asimismo, Laurence y Hull (2009) en su libro "El principio de Peter" parecen reforzar el concepto de CEO out of touch, ya que afirman que muchos empleados ejecutivos de todas las organizaciones han llegado a su nivel de incompetencia y en algún momento todos lo harán, y las organizaciones, rara vez, despiden a personas incompetentes.

Por otro lado, los autores también han observado una práctica común en empresas, principalmente americanas, de asignar actividades o tareas comunes entre jefes y subordinados como vínculo para desarrollar un trabajo colaborativo. Esta práctica constituiría un vehículo que ayudaría a reducir las características del gerente fuera de la realidad o síndrome de la arrogancia, y así mejorar la comunicación entre jefes y subordinados.

Como puede apreciarse, el fenómeno descrito como gerente fuera de la realidad se puede entrelazar con otros fenómenos como el manejo del poder, la incapacidad del líder o incluso rasgos de personalidad como el narcisismo.

En el contexto de trabajo colaborativo, el término actividades comunes se refiere a un trabajo que debe hacerse o emprenderse (Oxford, 2002). Asimismo, la idea de una comunicación organizacional está relacionada al proceso de creación y negociación de sistemas de significados colectivos y coordinados a través de prácticas simbólicas orientadas al logro de objetivos organizativos (Mumby y Kuhn, 2019).

La presente investigación encuentra también su justificación teórica en la Teoría Crítica de comunicación en las organizaciones, la misma que examina las prácticas de comunicación en las organizaciones que socavan la toma de decisiones plenamente representativas y, por tanto, reducen la calidad, la innovación y la equidad de la política de la empresa (Griffin, 2012).

En este contexto, toma relevancia el hecho de contar con la práctica de - compartir actividades - para así incrementar la calidad de la comunicación entre jefe y subordinado, de manera que esta impulse un mejor trabajo colaborativo, una mejor comunicación y un mejor clima laboral dentro de la organización. 


\section{MATERIALES Y MÉTODOS}

Este estudio se ajustó al nivel de investigación descriptiva y al diseño descriptivo correlacional (Sánchez y Reyes, 2015).

La población estuvo integrada por la población de la ciudad de Trujillo, perteneciente a la población económicamente activa ocupada del año 2020 , cuya muestra fueron 384 personas.

Para medir la calidad de la comunicación, se midio a través del cuestionario de la calidad de la comunicación y sus dimensiones/factores, elaborado por Mellado (2005). Este instrumento tiene 35 ítems con opciones de respuestas elaboradas según técnica de Likert, específicamente como se detalla a continuación: muy poca medida (uno), poca medida (dos), regular medida (tres), gran medida (cuatro) y muy gran medida (cinco).

Los ítems están agrupados en siete factores o dimensiones, tales como: en el primer factor, sobre la percepción de la comunicación jefe/trabajador, se agrupan las preguntas de la 1 a la 6; en el factor 2, sobre la comunicación ascendente, se agrupan las preguntas de la 7 a la 13; en el factor 3, la percepción sobre la confiabilidad del tipo información recibida desde el jefe, agrupa las preguntas de la 14 a la 18; en el factor 4, sobre la exactitud de la comunicación descendente y percepción de la calidad, agrupa las preguntas de la 19 a la 24 ; en el factor 5 , sobre percepción de las oportunidades de influir en el jefe, agrupa las preguntas de la 25 a la 28; en el factor 6 , sobre percepción de la confiabilidad de la información que se da entre compañeros de trabajo, agrupa las preguntas de la 29 a la 30; y, finalmente, en el factor 7, sobre percepción de la comunicación que debe haber entre subordinados, agrupa las preguntas de la 31 a la 35 .

En lo que refiere a la validez del cuestionario, se realizó mediante el método de juicio de expertos. Para ello se contó con el apoyo de tres especialistas en el tema, quienes otorgaron valoraciones equivalentes al 91\%, estimándose así, un grado de concordancia alto que sobrepasa el mínimo establecido por Guilford (1954); por lo tanto, se afirma que el instrumento fue válido para su aplicación a la muestra de estudio. Además, respecto a la confiabilidad, se obtuvo un alfa de 0.885 del total de los 35 ítems que componen los 11 factores de la variable.

Para medir las actividades comunes se utilizó un instrumento propio que fue elaborado por el equipo de investigación. Consta de 3 ítems con opciones de respuesta elaboradas según técnica de Likert, específicamente como se detalla a continuación: en muy poca medida, en poca medida, en regular medida, en gran medida y en muy gran medida. Los ítems están agrupados en tres factores o dimensiones, tales como: Similitud ( 1 ítem), Carga Laboral (1 ítem) y Expertise (1 ítem).

En lo que refiere a la validez del cuestionario, se realizó mediante el método de juicio de expertos, para ello se contó con el apoyo de tres especialistas en el tema, quienes otorgaron valoraciones equivalentes al $89 \%$, estimándose así un grado de concordancia alto que sobrepasa el mínimo establecido por Guilford (1954); por lo tanto, se afirma que el instrumento fue válido para aplicarlo a la muestra de estudio. Además, respecto a la confiabilidad, se obtuvo un alfa de 0.690 del total de los 3 ítems que componen las 3 dimensiones de la variable.

En consecuencia, estos resultados permiten concluir que la escala se mantiene en los ítems de cada una de sus dimensiones, una consistencia interna aceptable; mientras que, a escala global, presenta un alto nivel de consistencia interna, en virtud de lo cual califica como un instrumento confiable e idóneo para su administración a la muestra de investigación.

\section{RESULTADOS}

\section{Calidad de la comunicación}

Se observa en la tabla 1 que el promedio de todos los factores es $43.5 \%$ de los trabajadores encuestados, quienes presentan una percepción en nivel alto de la calidad de la comunicación. Asimismo, el $47.3 \%$ de los trabajadores encuestados presentan una percepción en un nivel medio y finalmente el 9.2\% de los trabajadores encuestados presentan una percepción en un medio bajo.

\section{Actividades comunes}

Se advierte en la tabla 2 que, de acuerdo con las respuestas obtenidas, las actividades comunes en promedio alcanzan el nivel alto en un $62.1 \%$. Respecto a la dimensión "carga laboral", esta se ubica en un nivel más alto con el 68.5\%; mientras que la dimensión "similitud" tiene un $65.4 \%$ y la dimensión "expertise" un 52.3\%.

\section{Relación entre calidad de la comunicación y actividades comunes}

Para la correlación de las variables en estudio, se obtuvo un coeficiente de Spearman $(r=0,619)$ altamente significativo con $\mathrm{p}<0,01$ (tabla 3 ); resultado que señala que entre ambas variables existe una relación directa y significativa, con resultados cercanos a una correlación fuerte (Elorza, 2008). Al ser la relación positiva, entonces se puede señalar que, a mayor cantidad de actividades comunes, mejor calidad en la comunicación jefe-subordinado. 
Tabla 1

Resultados descriptivos en frecuencias y porcentajes sobre la calidad de la comunicación y sus dimensiones

\begin{tabular}{|c|c|c|c|c|c|}
\hline \multirow{2}{*}{ Variable/Dimensión } & \multirow{2}{*}{ Estadístico } & \multicolumn{3}{|c|}{ Nivel } & \multirow{2}{*}{ Total } \\
\hline & & Alto & Medio & Bajo & \\
\hline \multirow{2}{*}{ "Factor 1" } & $\mathrm{F}$ & 220 & 120 & 44 & 384 \\
\hline & $\%$ & $57.3 \%$ & $31.3 \%$ & $11.5 \%$ & $100 \%$ \\
\hline \multirow{2}{*}{ "Factor 2" } & $\mathrm{F}$ & 208 & 143 & 33 & 384 \\
\hline & $\%$ & $54.2 \%$ & $37.2 \%$ & $8.6 \%$ & $100 \%$ \\
\hline \multirow{2}{*}{ "Factor 3" } & $\mathrm{F}$ & 156 & 184 & 44 & 384 \\
\hline & $\%$ & $40.6 \%$ & $47.9 \%$ & $11.5 \%$ & $100 \%$ \\
\hline \multirow{2}{*}{ "Factor 4" } & $\mathrm{F}$ & 132 & 230 & 22 & 384 \\
\hline & $\%$ & $34.4 \%$ & $59.9 \%$ & $5.7 \%$ & $100 \%$ \\
\hline \multirow{2}{*}{ "Factor 5" } & $\mathrm{F}$ & 127 & 225 & 32 & 384 \\
\hline & $\%$ & $33.1 \%$ & $58.6 \%$ & $8.3 \%$ & $100 \%$ \\
\hline \multirow{2}{*}{ "Factor 6" } & $\mathrm{F}$ & 130 & 215 & 39 & 384 \\
\hline & $\%$ & $33.9 \%$ & $56.0 \%$ & $10.2 \%$ & $100 \%$ \\
\hline \multirow{2}{*}{ "Factor 7" } & $\mathrm{F}$ & 197 & 154 & 33 & 384 \\
\hline & $\%$ & $51.3 \%$ & $40.1 \%$ & $8.6 \%$ & $100 \%$ \\
\hline \multicolumn{2}{|c|}{ Promedio } & $43.5 \%$ & $47.3 \%$ & $9.2 \%$ & $100 \%$ \\
\hline
\end{tabular}

Fuente: Elaboración propia

Tabla 2

Resultados descriptivos en frecuencias y porcentajes sobre las actividades comunes y sus dimensiones

\begin{tabular}{|c|c|c|c|c|c|}
\hline \multirow{2}{*}{ Variable/Dimensión } & \multirow{2}{*}{ Estadístico } & \multicolumn{3}{|c|}{ Nivel } & \multirow{2}{*}{ Total } \\
\hline & & Alto & Medio & Bajo & \\
\hline \multirow{2}{*}{ Similitud } & $\mathrm{F}$ & 251 & 68 & 65 & 384 \\
\hline & $\%$ & $65.4 \%$ & $17.7 \%$ & $16.9 \%$ & $100.0 \%$ \\
\hline \multirow{2}{*}{ Carga laboral } & $\mathrm{F}$ & 263 & 53 & 68 & 384 \\
\hline & $\%$ & $68.5 \%$ & $13.8 \%$ & $17.7 \%$ & $100.0 \%$ \\
\hline \multirow{2}{*}{ Expertise } & $\mathrm{F}$ & 201 & 96 & 87 & 384 \\
\hline & $\%$ & $52.3 \%$ & $25.0 \%$ & $22.7 \%$ & $100.0 \%$ \\
\hline \multicolumn{2}{|c|}{ Promedio } & $62.1 \%$ & $18.8 \%$ & $19.1 \%$ & $100.0 \%$ \\
\hline
\end{tabular}

Fuente: Elaboración propia

Tabla 3

Resultados de correlación de Spearman de las variables Actividades Comunes y Calidad de la Comunicación

\begin{tabular}{llc}
\hline Calidad de la comunicación & & \\
\hline \multirow{3}{*}{ Actividades comunes } & Correlación de Spearman & $0,619^{* *}$ \\
& Sig. (unilateral) & 0,000 \\
& $\mathrm{~N}$ & 384 \\
\hline
\end{tabular}

** Significativo al nivel de $\mathrm{p}<0,01$.

Fuente: Elaboración propia 


\section{DISCUSIÓN}

El objetivo de esta investigación fue descubrir si el mantener actividades comunes entre jefes y subordinados mejoraría la comunicación entre estos. Las actividades comunes cumplen el papel de mantener al jefe en contacto con la realidad del subordinado, lo cual tiene como efecto el mejorar la calidad de comunicación entre ambos. Este resultado es consecuente con el estudio de Owen (2012), cuya conclusión es que el poder tiende a corromper a las personas dado que genera una distorsión de la realidad.

Asimismo, los resultados de este estudio son compatibles con los estudios del profesor Sapolsky (2005), los cuales evidencian que al reducir la distancia social entre primates estos se pueden comunicar mejor. De manera similar ocurre con los humanos al crear un contacto cercano entre ellos, lo que indicaría una alta probabilidad de mejoría en la comunicación entre jefes y sus subordinados.

El presente estudio apunta a verificar y recomendar la asignación de tareas comunes entre jefes y subordinados como una herramienta para mejorar la comunicación medio por el cual se evidencia o percibe al gerente fuera de la realidad - y aminorar o eliminar sus efectos.

Esta investigación puede ser el primer eslabón para una práctica recomendada en las organizaciones que busquen mejorar su comunicación interna, mejorar la coordinación para un trabajo colaborativo y puedan solucionar problemas comunes. Finalmente, esta investigación puede dar inicio una investigación sobre los niveles del síndrome de Hubris (síndrome de la arrogancia) que se presentan en los puestos altos de las empresas en el país.

\section{REFERENCIAS}

Alvesson, M., y Spicer, A. (2016). The Stupidity Paradox. London: Profile Books.

Banaji, Bazerman, y Chugh. (2003). How (un) ethical are you? USA: Harvard Business Review.

Elorza, H. (2008). Estadística para las ciencias sociales, del comportamiento y de la salud. Recuperado de: https://www.uv.mx/rmipe/files/2015/09/Estadistica-para-las-ciencias-sociales-del-comportamiento-y-de-la-salud.pdf
Griffin, E. (2012). A first look at communication theory. New York: McGraw-Hill.

Guilford, J. P. (1954). Psychometric methods. New York: McGraw-Hill.

Laurence, J.P. y Hull, R. (2009). The Peter Principle. Why things always go wrong. USA: Harper Collins e-books.

Mellado, C. (2005). Validez de Constructo de una Escala para Medir Clima y Liderazgo desde una Óptica Comunicativa en la Pequeña Empresa Industrial. Razón y Palabra, (47). Recuperado de: https://www.redalyc. org/articulo.oa? $\mathrm{id}=199520655010 \&$ idp $=1 \& \mathrm{cid}=66200$

Mumby, D., y Kuhn, T. (2019). Organizational Communication. A Critical Introduction. California: SAGE Publications.

Oxford. (2002). American College Dictionary. New York: Oxford University Press.

Owen, D. (2012). The Hubris Syndrome. Bush, Blair and the Intoxication of Power. New York: Methuen \& Co.

Owen, D., y Davidson, J. (2009). Hubris syndrome: An acquired personality disorder? A study of US Presidents and UK Prime Ministers over the last 100 years. Brain, 132(5), 1396-1406. https://doi.org/10.1093/brain/ awp008

Roberts, N. (20 de octubre de 2017). The CEO out of touch. Recuperado de: https://www.linkedin.com/pulse/out-of-touch-ceo-nancy-roberts/

Sánchez, H. y Reyes, C. (2015). Metodología y diseños en la investigación científica. Lima: Business Support Aneth.

Sapolsky, R. (2005). The Influence of Social Hierarchy on Primate Health. Science, 308(5722), 648-652. https:// doi.org/10.1126/science. 1106477 
